7

\title{
Early Patellofemoral Cartilage and Bone Degeneration in a Rat Model of Noninvasive
}

\section{Anterior Cruciate Ligament Rupture}

${ }^{1}$ Samantha E. Hartner, MS; ${ }^{1}$ Michael D. Newton, MS; ${ }^{1}$ Mackenzie M. Fleischer, MS; ${ }^{1,2}$ Kevin C. Baker, PhD; ${ }^{3}$ Tristan Maerz, PhD*

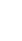

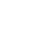

\author{
${ }^{1}$ Orthopaedic Research Laboratory, Beaumont Health, Royal Oak, MI \\ ${ }^{2}$ Department of Orthopaedic Surgery, Oakland University - William Beaumont School of \\ Medicine, Rochester, MI \\ ${ }^{3}$ Department of Orthopaedic Surgery, University of Michigan, Ann Arbor, MI
}

*Corresponding Author:

Tristan Maerz, PhD

Department of Orthopaedic Surgery, University of Michigan

109 Zina Pitcher Place, Ann Arbor MI 48109

Phone: 734936 2566; E-Mail: tmaerz@umich.edu

Running Title: Patellofemoral OA after ACL Rupture

\section{Author contributions}

All authors have made substantial contributions to the conception and design of the study, acquisition of data, and/or analysis and interpretation of data, as well as manuscript preparation and final approval of the submitted manuscript. TM takes responsibility for the integrity of the work as a whole. Investigation performed at the Orthopaedic Research Laboratories of Beaumont Health in Royal Oak, Michigan. 


\section{ABSTRACT}

25 Background: Anterior cruciate ligament rupture (ACLR) is a well-known risk factor for the

26 development of post-traumatic osteoarthritis (PTOA). While clinical and pre-clinical studies

27 have characterized the onset and progression of PTOA in the tibiofemoral joint compartment,

28 very little is known about degenerative changes in the patellofemoral compartment after ACL

29 injury.

30 Hypothesis/Purpose: To evaluate the extent to which ACL rupture induces acute patellofemoral

31 joint degeneration by quantifying articular cartilage morphology and remodeling of subchondral

32 and trabecular bone microarchitecture in the patellofemoral compartment.

33 Study Design: Descriptive laboratory study.

34 Methods: Adult female Lewis rats were randomized to undergo either a non-surgical ACL

35 rupture or a Sham procedure ( $\mathrm{n}=6$ per group). Ex vivo contrast-enhanced micro-computed

36 tomography $(\mu \mathrm{CT})$ and histological evaluation of the patellofemoral compartment were

37 performed at 2-weeks post-injury, representing a timepoint of documented early PTOA in the

38 tibiofemoral compartment in this model.

39 Results: ACL rupture causes osteophyte formation in the patella and mild degeneration in the superficial zone of articular cartilage (AC), including surface fibrillation, fissures, increased

41 cellularity, and abnormal chondrocyte clustering at two weeks post-injury. Contrast-enhanced

$42 \mu \mathrm{CT}$ analysis demonstrates significant increases in $\mathrm{AC}$ thickness of patellar and trochlear

43 cartilage. Loss of subchondral bone thickness, bone volume fraction, and tissue mineral density,

44 as well as changes to trabecular microarchitecture in both the patella and trochlea, were

45 indicative of catabolic bone remodeling. 
46 Conclusion: These results demonstrate that the patellofemoral joint develops mild but evident

47 degenerative changes in the acute time period following ACL rupture, extending the utility of

48 this rat model to the study of degenerative patellofemoral changes following joint trauma.

49 Clinical Relevance: ACL rupture causes mild degeneration and swelling of articular cartilage,

50 coupled with catabolic bone remodeling in the patellofemoral compartment. Characterizing the

51 pathophysiology of patellofemoral PTOA in its early stages may provide a better understanding

52 of disease progression and provide opportunities for preventative therapeutic intervention.

53

54 Key Terms: post-traumatic osteoarthritis; patellofemoral cartilage; bone microarchitecture;

55 anterior cruciate ligament rupture

56 


\section{INTRODUCTION}

Anterior cruciate ligament (ACL) rupture is one of the most common soft-tissue musculoskeletal injuries and a well-known risk factor for post-traumatic osteoarthritis $(\mathrm{PTOA})^{4,24}$. The progression of tibiofemoral PTOA following ACL rupture has been well-

61 characterized in both clinical and pre-clinical literature ${ }^{6,22,25,41}$. However, comparatively few

62 studies have characterized patellofemoral compartment OA (PFOA) following ACL rupture, and relatively little is known regarding incidence and overall phenotypic manifestation of joint degeneration in this compartment. Recent work suggests that PFOA may be equally or even more prevalent than tibiofemoral OA: Culvenor et al demonstrate that within 5-10 years postACL rupture, $47 \%$ of patients exhibit PFOA progression - including osteophytes, cartilage

67 lesions, and joint space narrowing - compared to a $31 \%$ incidence of tibiofemoral $\mathrm{OA}^{9}$. Furthermore, recent MRI-based assessment of PFOA indicated that $40 \%$ of patients exhibit detectable patellofemoral cartilage loss within 5 years of ACL injury, with cartilage thickness in the trochlea decreasing by as much as $1 \%$ annually in the first 2 years $^{8}$.

72 acute manifestations of PFOA and the associated microstructural, cellular, and molecular

73 changes following ACL injury remain poorly understood. Clinical evidence regarding the nature

74 of early patellofemoral tissue changes is lacking, as most clinical studies of PFOA track joint

75 degeneration once disease has already developed. Natural history studies assessing PFOA

76 incidence and development commonly employ radiography ${ }^{9,20,32,34}$, which can diagnose joint

77 space narrowing and osteophyte formation but is inherently incapable of assessing the extent and

78 location of articular cartilage and bone degeneration, especially in early disease. While MRI is

79 the gold-standard for assessment of cartilage thickness and bone marrow lesions ${ }^{7,8,21}$, it lacks 


8

imaging resolution to measure injury-induced changes to bone microarchitecture. Thus, the collective understanding of acute PFOA following ACL injury is currently limited.

Small animal models of ACL injury have become invaluable tools for the preclinical study of PTOA. In a previous rat study of surgical ACL transection, decreased subchondral bone (SCB) perfusion and associated cartilage and bone lesions were observed in the patellofemoral compartment at intermediate and chronic time points following injury ${ }^{40}$, demonstrating chronic manifestations of ACL injury in the rodent patellofemoral compartment. However, this study did not analyze acute time points to understand early disease, and surgical models of ACL injury are inherently limited in their assessment of acute injury due to the confounding influence of surgical trauma and lack of mechanical loading. Non-invasive models of isolated ACL rupture, whereby joint injury is induced mechanically, provide a highly controlled and clinically-representative means of inducing joint injury, and the utilization of these models to study tibiofemoral PTOA has increased in recent years $3,6,23,26,38$. However, to date, the extent of patellofemoral remodeling and degeneration in these non-invasive injury models remains largely unexplored, and it is unclear to what extent these models may be employed to study PFOA pathophysiology and potential treatments. To this end, the aim of this study was to employ both quantitative imaging and histological evaluation to characterize acute changes to bone and articular cartilage within the patellofemoral joint in a rat model of non-invasive ACL rupture.

\section{MATERIALS AND METHODS}

\section{Rats and Non-Invasive ACL Rupture}

Under an IACUC-approved protocol, 12 female Lewis rats (aged 14 weeks, weight 200220g, Charles River Laboratories, Wilmington, MA) were randomized to either non-invasive 
103 ACL rupture (ACLR) or Sham groups ( $n=6$ per group). The sample size was determined based 104 on published $\mu \mathrm{CT}$ data from the same $\operatorname{model}^{25}$ : given the previously-observed effect size in 105 trabecular bone volume fraction between control rats and ACLR rats (a morphometric parameter 106 most responsive to injury and an outcome we deemed most applicable to the present study) in 107 order to detect a $5 \%$ difference between groups, (effect size $=2.2, \alpha=0.05$, power $=0.9$ ), six rats 108 would be required. To induce ACLR or Sham injury, rats were placed prone on a materials 109 testing system (Insight 5, MTS, Eden Prairie, MN) with the right knee positioned in $100^{\circ}$ of

110 flexion and the hindpaw held in $30^{\circ}$ of dorsiflexion in a custom fixture. In the ACLR group,

111 ACL rupture was induced by applying a preload, ten preconditioning cycles, and a subsequent 112 rapid compressive displacement to the knee joint, as described previously ${ }^{26}$. The Sham group

113 was subjected to preload and preconditioning cycles only. Anesthesia was induced by

114 intraperitoneal ketamine and xylazine, and maintained under 1\%-2\% inhaled isoflurane. All rats

115 were administered $5 \mathrm{mg} / \mathrm{kg}$ subcutaneous carprofen immediately prior to Sham/ACLR injury, 116 and buprenorphine $(1.2 \mathrm{mg} / \mathrm{kg})$ was administered for analgesia at $12 \mathrm{~h}$ and $24 \mathrm{~h}$ post-procedure.

117 All procedures were completed in the morning, and rats individually housed and allowed $a d$

118 libitum cage activity and access to standard rodent chow under a 12-hour light/dark cycle for 2

119 weeks post-procedure until euthanasia via $\mathrm{CO}_{2}$ asphyxiation. To perform contrast-enhanced 120 imaging, femora and patellae were completely dissected to expose the articular surface, fixed in $12110 \%$ neutral buffered formalin for 48 hours, and transferred to $70 \%$ ethanol for long-term 122 storage. 
To facilitate contrast-enhanced $\mu \mathrm{CT}$ of articular cartilage, femora and patellae were

126

127

128

129

130

131

132

133

134

135

136

137

138

139

140

141

142

143

144

145

146

147 rehydrated in PBS overnight and incubated in 20\% v/v ioxaglate (Hexabrix 320, Guerbet LLC, Princeton, NJ) for 2 hours prior to $\mu \mathrm{CT}$ imaging as previously described ${ }^{36}$. In preliminary experiments, a 2-hour contrast agent incubation was found to be sufficient to achieve equilibrium in articular cartilage (data not shown). Both patellae and femora were securely positioned in custom sample holders for consistent imaging positioning and orientation, and humidifying silica beads were used to maintain a $70 \%$ humid environment during $\mu \mathrm{CT}$ acquisition. Femora were scanned on a $\mu$ CT-40 (Scanco Medical AG, Brüttisellen, Switzerland) at 55 kVp, $145 \mu$ A, 300 $\mathrm{ms}$ integration time, with an isotropic voxel size of $8 \mu \mathrm{m}$. Patellae were scanned on a VivaCT-80 (Scanco Medical AG) at $55 \mathrm{kVp}, 145 \mu \mathrm{A}, 800 \mathrm{~ms}$ integration time, with a $12 \mu \mathrm{m}$ isotropic voxel size. The use of two imaging systems was necessary given study timeline restrictions, and validation data demonstrates equivalent morphometry results between these two imaging resolutions (data not shown). Furthermore, no comparisons are made between the two imaging protocols. Following scanning, specimens were washed in PBS for 30 minutes and graded back to $70 \%$ ethanol.

\section{Imaging Analysis}

Femoral and patellar articular cartilage, subchondral bone, and trabecular bone volumes of interest were isolated using an automated, atlas registration-based segmentation algorithm in MATLAB (Mathworks, Natick, MA), as previously described ${ }^{33}$. All segmentations were reviewed for accuracy by an experienced investigator. Femoral AC and bone volumes were further segmented to isolate the trochlear compartment, defined as all bone and AC proximal to the femoral notch. AC was quantitatively analyzed via mean thickness (AC.Th) using the direct- 
148 distance transform (i.e "sphere-fitting") method in boneJ". Trabecular bone was assessed via

149 bone volume fraction (BV/TV), tissue mineral density (TMD), trabecular thickness (Tb.Th),

150 trabecular spacing (Tb.Sp), and trabecular number (Tb.N) according to standard methods ${ }^{2}$.

151 Subchondral bone was assessed via subchondral bone thickness (SCB.Th), BV/TV, and TMD. A

152 standardized hydroxyapatite phantom was used to calibrate imaging data to mineral density.

154 Histology

Patellae, including the attached quadriceps and patellar tendons, were decalcified, embedded in paraffin and bisected sagittally, and a single $5 \mu \mathrm{m}$ sagittal section (including both halves) was taken. Femora underwent additional histological assessments of the femoral 158 condyles as part of a separate study on the tibiofemoral compartment which required the use of non-decalcified methods ${ }^{28}$. As such, femora were embedded in methyl methacrylate and bisected 160 into medial and lateral halves. Two $5 \mu \mathrm{m}$ sections were taken, spaced $200 \mu \mathrm{m}$ apart. For this 161 study, trochlear analysis was carried out on lateral compartment sections, which fully 162 encompassed the trochlear articular cartilage. All sections of both patellae and femora were 163 stained with Safranin-O/Fast Green and imaged at 20x magnification using a slide imaging 164 system (Aperio, Leica 122 Biosystems, Buffalo Grove, IL). Two blinded raters scored each 165 section using the Osteoarthritis Research Society International (OARSI) Modified Mankin 166 score $^{16}$, and graders conferred on disparate scores (differences of greater than one point) until 167 consensus was reached. Histologic scores were averaged across all sections for each limb, and an 168 aggregate group average was calculated. 
Statistical analysis was performed using SPSS (IBM, Armonk, NY). The normality and

172 equal variance assumptions were confirmed for continuous data. $\mu \mathrm{CT}$ data were assessed via

173 mixed two-way analysis of variance (ANOVA) with one within-subject factor (Injured vs

174 Contralateral) and one between-subject factor (ACLR vs Sham). Histologic grades were assessed

175 via the Kruskal Wallis test, with post hoc comparisons via Mann-Whitney U tests with a

176 Bonferroni correction for multiple comparisons. Adjusted P values below 0.05 were considered

177 significant.

178

179

180

181

182

183

184

185

186

187

188

189

190

191

192

193

\section{RESULTS}

All animals maintained good health status for the 2-week period following the ACLR and Sham procedures and thus all 6 rats from both experimental groups were included in the analysis.

\section{Articular Cartilage Exhibits Acute Swelling Following ACLR}

AC was significantly thicker in the ACLR Injured limb compared to the Contralateral limb in the patella and trochlea (Patella $-12.3 \%$ increase, $P=0.003$; Trochlea $-8.7 \%$ increase, $P=0.005)$, and compared to the Sham Uninjured limb in the patella $(18.8 \%$ increase, $P=0.001)$

(Figure 1). Interestingly, patellar AC of the Sham Uninjured limb was significantly thinner compared to the Sham Contralateral ( $8.1 \%$ decrease, $P=0.026)$, indicating a subtle effect of sham loading. In the patella of the ACLR Injured limb, AC thickening occurred over the entire superior cartilage surface, encompassing both the medial and lateral regions, while in the Sham Uninjured limb, cartilage thinning was most pronounced on the superior and lateral surfaces

(Figure 1A). In the trochlea of the ACLR Injured limb, AC thickening occurred primarily in the proximal aspect but with reduced magnitude compared to the patella (Trochlea: $8.7 \%$ increase; 
194 Patella: $12.3 \%$ increase). Taken together, these findings demonstrate that ACLR induces acute

195 swelling/thickening of both patellar and trochlear articular cartilage, with a greater magnitude of

196 change observed in the patella.

197

Injury-Induced Loss of Trabecular and Subchondral Bone is Observed in the Patella and

Trochlea

ACLR induced catabolic trabecular and subchondral bone remodeling of both the patella

201

202

203

204

205

206

207

208

209

210

211

212

213

214

215

216

and the trochlea. In the patella, trabecular BV/TV, TMD, Tb.Th, and Tb.N were significantly decreased and Tb.Sp was significantly increased in the ACLR Injured limb compared to the Contralateral limb (BV/TV: $12.9 \%$ decrease, $P<0.001$, TMD: $2.3 \%$ decrease, $P=0.001$, Tb.Th:

6.8\% decrease, $P<0.001$, Tb.N: $6.5 \%$ decrease, $P<0.001$, Tb.Sp: $10.1 \%$ increase, $P<0.001)$ and compared to the Sham Uninjured limb (BV/TV: 12.7\% decrease, $P<0.001$, TMD: $2.0 \%$ decrease, $P=0.046$, Tb.Th: $6.4 \%$ decrease, $P=0.022$, Tb.N: $6.8 \%$ decrease, $P=0.001$, Tb.Sp: $10.8 \%$ increase, $P<0.001$ ) (Figure 2). In the trochlea, epiphyseal Tb.Th was significantly decreased in the ACLR Injured limb compared to both the Contralateral (4.3\% decrease, $P=$ $0.008)$ and Sham Uninjured limbs $(6.2 \%$ decrease, $P=0.002)$. Epiphyseal BV/TV was significantly lower in the ACLR Injured limb compared to the Sham Uninjured limb (7.3\% decrease, $P=0.023)$. In the subchondral compartment, subchondral BV/TV and thickness were significantly decreased in the ACLR Injured limb compared to the Contralateral limb (Patella BV/TV: $3.6 \%$ decrease, $P=0.001$, SCB.Th: $12.0 \%$ decrease, $P<0.001$; Trochlea - BV/TV: 2.5\% decrease, $P<0.001$, SCB.Th: $11.5 \%$ decrease, $P<0.001)$ and compared to the Sham Uninjured limb (Patella - BV/TV: $4.2 \%$ decrease, $P<0.001$, SCB.Th: $15.0 \%$ decrease, $P=$ 0.002; Trochlea - BV/TV: $2.9 \%$ decrease, $P=0.002$, SCB.Th: $15.7 \%$ decrease, $P=0.001$ ) 
217 (Figure 2). Although no formal region-dependent analysis was performed, loss of SCB.Th

218 appeared to occur globally across the patella and trochlea in the ACLR limb, while in the Sham

219 limb, increased SCB.Th appears more pronounced on the inferior patella and medial trochlea.

220 Patellar SCB TMD was significantly decreased in the ACLR Injured limb compared to the

221 Contralateral limb (3.0\% decrease, $P<0.001)$. SCB.Th of the Sham Uninjured limb was

222 significantly higher compared to the Sham Contralateral limb (Patella: $5.1 \%$ increase, $P=0.014$,

223 Trochlea: $5.6 \%$ increase, $P=0.010)$. Taken together, these results demonstrate the acute loss of

224 both trabecular and subchondral bone in the patellofemoral compartment following ACLR.
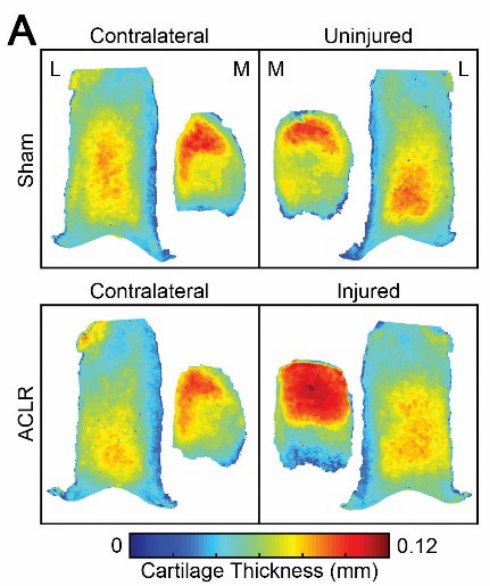

B Cartilage Thickness

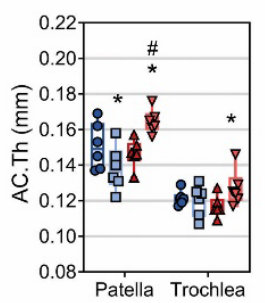

- Sham Contralateral $\triangle A C L R$ Contralateral

口Sham Uninjured $\quad \nabla$ ACLR Injured

Figure 1. Three-dimensional articular cartilage thickness maps (A) and numerical thickness

227 results $(B)$ derived from contrast-enhanced $\mu C T$ demonstrate thickening of both patellar and trochlear cartilage following ACLR. The top of the figure represents the superior/proximal aspect of the patella and trochlea. $n=6$ for all groups. * represents significant difference

230 compared to Contralateral limb $\left({ }^{*} P<0.05,{ }^{* *} P<0.001\right)$; \# represents significant difference

231 compared to Sham Uninjured limb $\left({ }^{\#} P<0.05,{ }^{\# \#} P<0.001\right)$; mixed two-way ANOVA. 
A

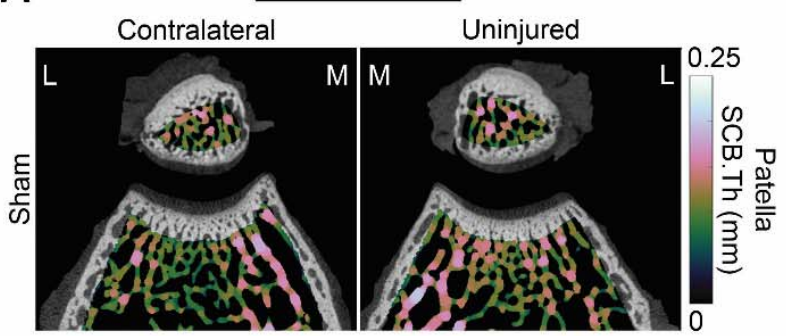

Contralateral
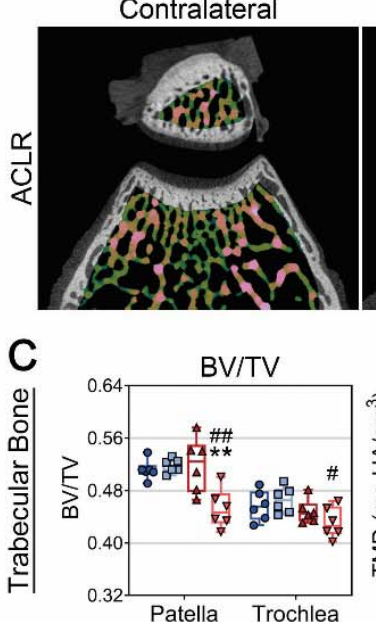

D

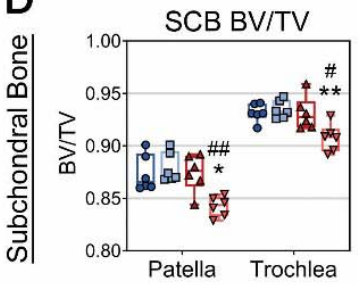

Injured

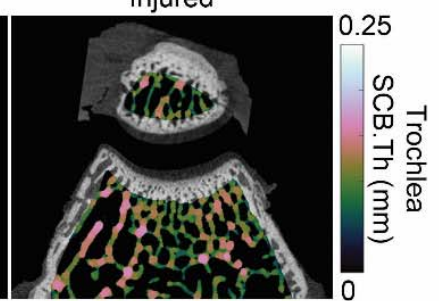

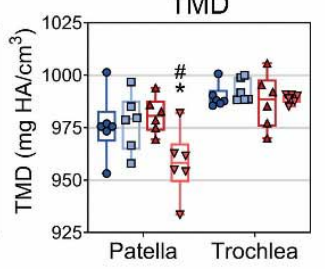

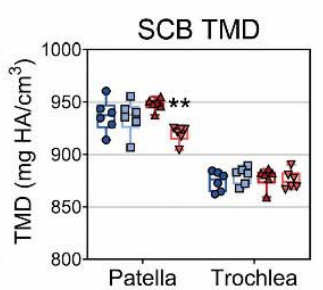

B

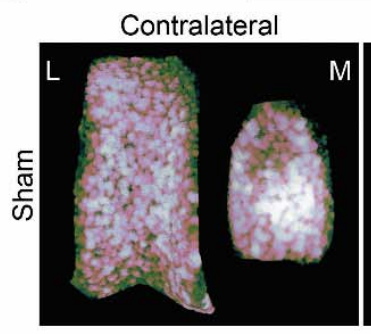

Contralateral

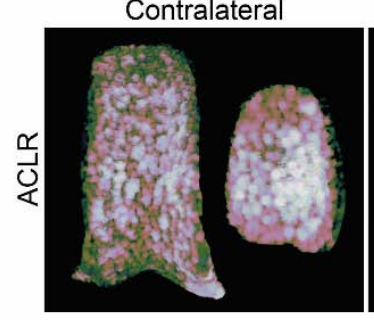

Uninjured

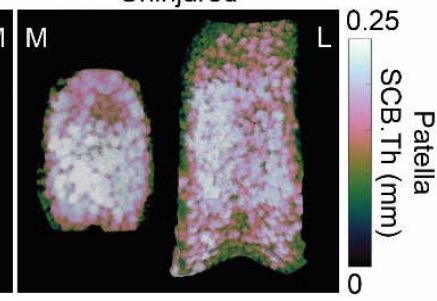

Injured

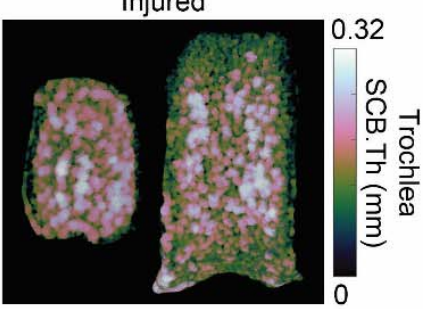

Tb.Th
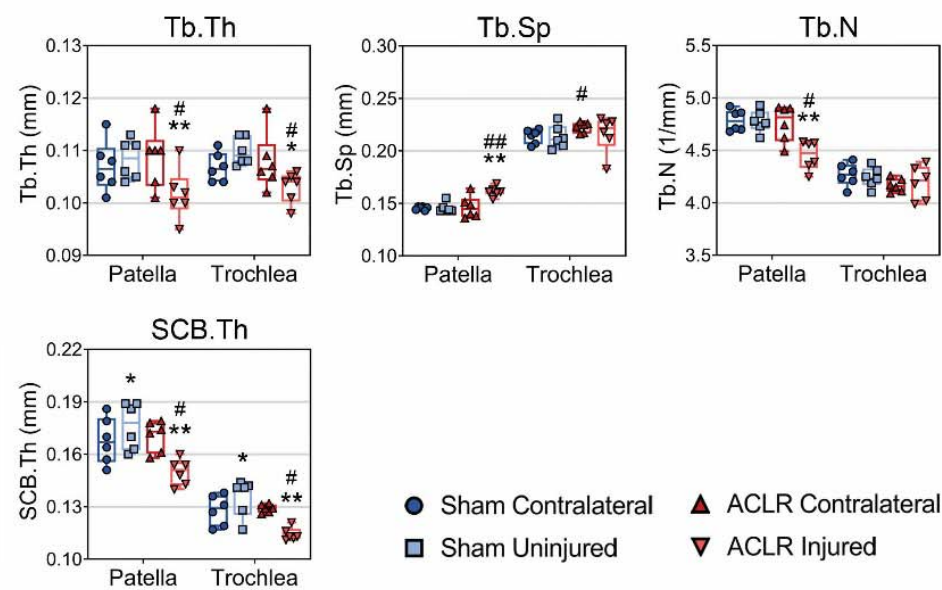

- Sham Contralateral

$\square$ Sham Uninjured $\triangle$ ACLR Contralateral

$\boldsymbol{\nabla}$ ACLR Injured

Figure 2. Trabecular bone thickness maps of the patella and trochlea overlaid onto

representative axial $\mu C T$ images show increased Tb.Sp and reduced Tb.Th and Tb.N in the 
241

242

243

244

245

246

247

248

249

250

251

252

253

254

255

256

257

258

259

260

261

262

263

represents significant difference compared to Sham Uninjured limb $\left({ }^{\#} P<0.05,{ }^{\# \#} P<0.001\right)$;

mixed two-way ANOVA.

Histology Demonstrates Superficial Articular Cartilage Damage, Osteophyte Formation, and Abnormal Chondrocyte Morphology Following ACL Rupture

Patellar and trochlear cartilage of both Sham limbs appeared normal, with a smooth articular surface, normal chondrocyte morphology, and homogenous Saf-O staining distributions. While our contrast-enhanced $\mu \mathrm{CT}$ results demonstrate mild thinning of Sham Uninjured cartilage in the patella, we did not observe any notable degenerative changes in Sham limbs on histology. ACLR Injured patellae demonstrate mild but evident degenerative histological changes, including surface fibrillation, reduction of intracellular and extracellular PG staining, increased chondrocyte clustering, and osteophyte formation (Figure 3A-B). We also observed an increased incidence of hypertrophic chondrocytes in deep cartilage, most notably at the superior patellar aspect where articular cartilage thickening was observed. In superficial patellar cartilage, a greater incidence of abnormal chondrocyte clustering was noted in ACLR Injured limbs (Figure

3A, red arrowheads). Superficial fissures and abnormal chondrocyte clustering were noted to be more prevalent in the inferior region of patellar cartilage. Osteophytes appeared immature, with primarily cartilaginous composition and focal regions of mineralized tissue, indicative of active osteophyte formation. This osteophyte formation was exclusively observed at the superior aspect of the patella (Figure 3B). Correspondingly, ACLR Injured patellae had a significantly higher OARSI score compared to both ACLR Contralateral $(P=0.015)$ and Sham Uninjured limbs $(P=$ 0.015), as well as several significantly higher sub-scores compared to both Contralateral (AC Structure: $P=0.018$, PG Staining: $P=0.014$, Cellularity: $P=0.008$, Osteophyte: $P=0.004)$ and 
264 Sham Uninjured limbs (AC Structure: $P=0.014$, PG Staining: $P=0.014$, Cellularity: $P=0.007$, 265 Osteophyte: $P=0.004)$ (Figure 3C).

266 ACLR Injured trochleae had largely intact cartilage surfaces, with only minor noted

267 superficial fibrillation (Figure 4A, green arrowhead). Notably, ACLR trochleae exhibited focal 268 regions of intense Saf-O staining and pockets of abnormal clustering/cloning in superficial-layer 269 cells. ACLR Injured trochleae had a significantly higher OARSI score compared to Sham

270 Uninjured limbs $(P=0.024)$. Multiple OARSI sub-scores were significantly higher in ACLR

271 trochleae compared to Sham Uninjured limbs (AC Structure: $P=0.038$, PG Staining: $P=0.022$,

272 Cellularity: $P=0.023$ ) and the tidemark sub-score was significantly higher compared to ACLR

273 Contralateral limbs $(P=0.035)$ (Figure 4B). Taken together, our histologic results demonstrate

274 that at this acute post-injury timepoint, ACL rupture induces mild articular cartilage degeneration

275 of the patella and trochlea, largely contained to the superficial zone, and osteophyte formation at 276 the superior aspect of the patella. 
A
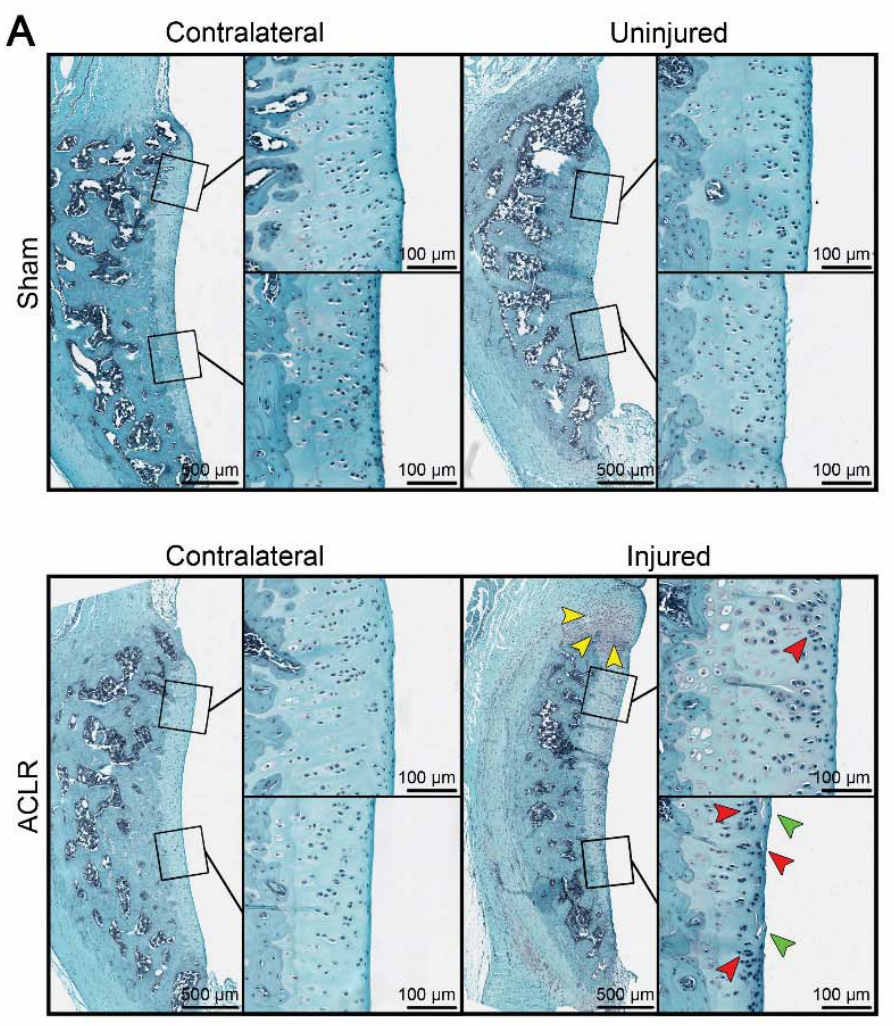

C
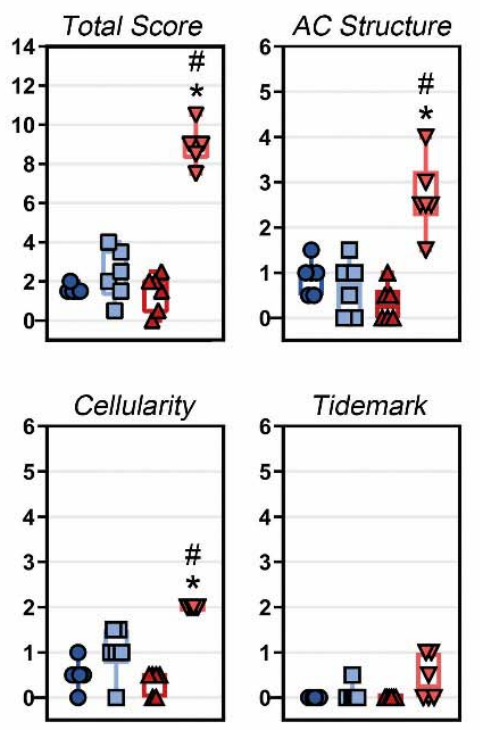
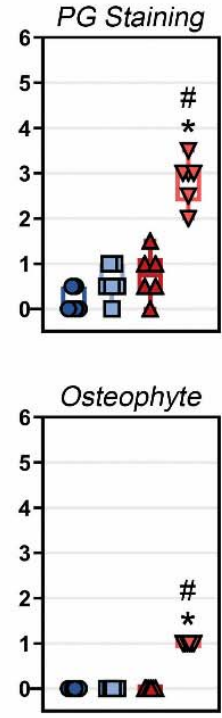

- Sham Contralateral

$\square$ Sham Uninjured

$\Delta$ ACLR Contralateral

$\nabla$ ACLR Injured

B

Osteophyte Formation
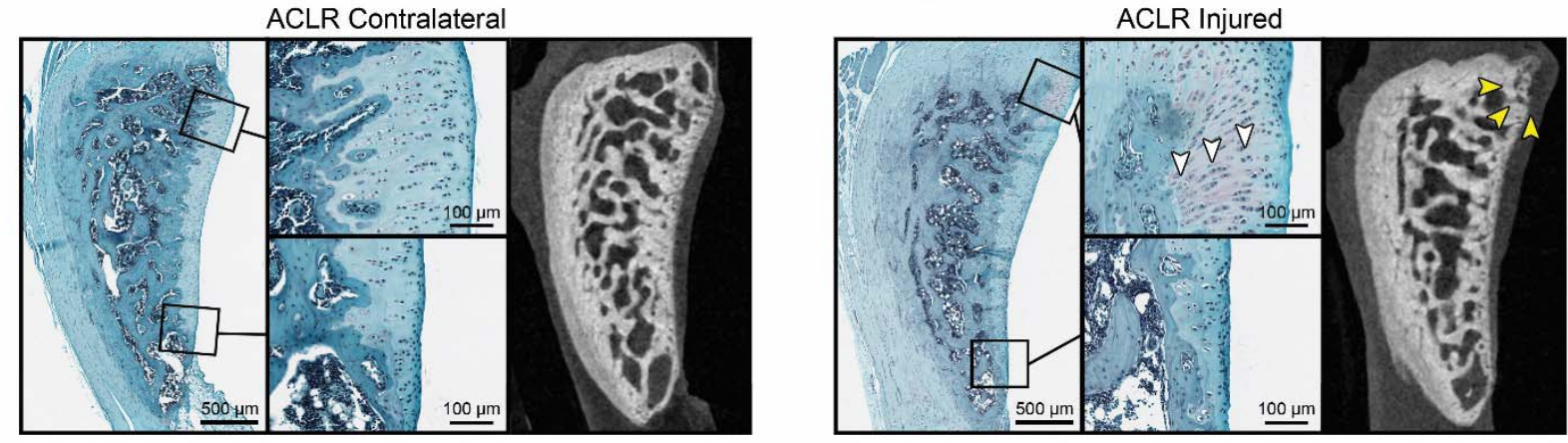

Figure 3. Representative sagittal histology of patellae demonstrates that ACLR Injured patellae

279 exhibit superficial fissures (green arrowheads), osteophyte formation (yellow arrowheads), and

abnormal chondrocyte morphology and clustering (red arrowheads) (A). Osteophyte formation

281 at the superior aspect of ACLR Injured patellae was evident on both histology and $\mu C T$.

282 Proliferation of hypertrophic and pre-hypertrophic chondrocytes indicative of endochondral

283 ossification is evident in superior aspect of the ACLR Injured patella (white arrowheads) (B).

284 ACLR Injured patellae exhibited significantly higher OARSI Modified Mankin scores and sub- 
Osteophyte - 3) (C). The top of the figure represents the superior/proximal aspect of the patella.

A

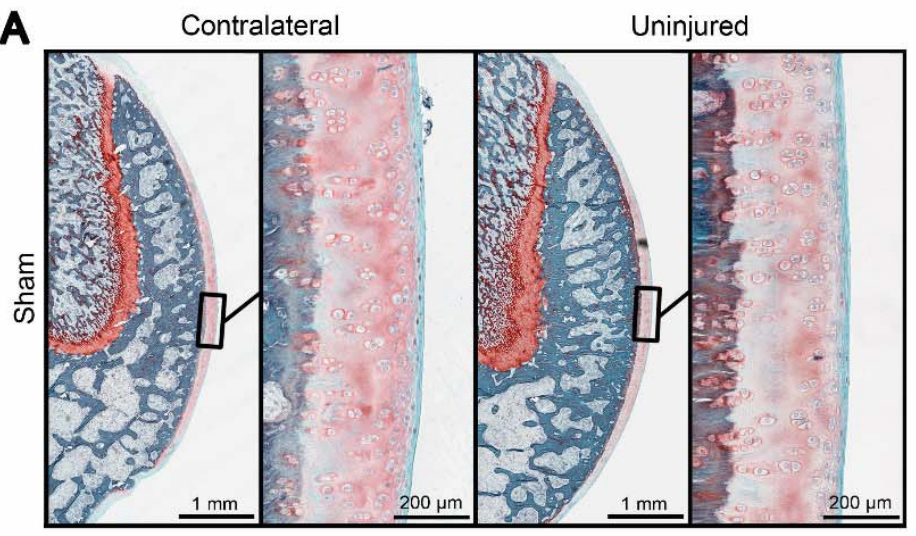

291

292

293

294

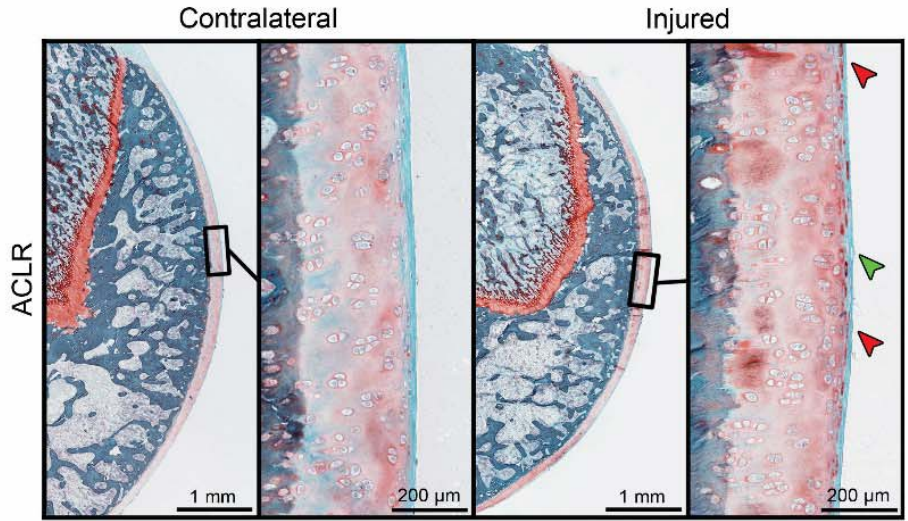

B
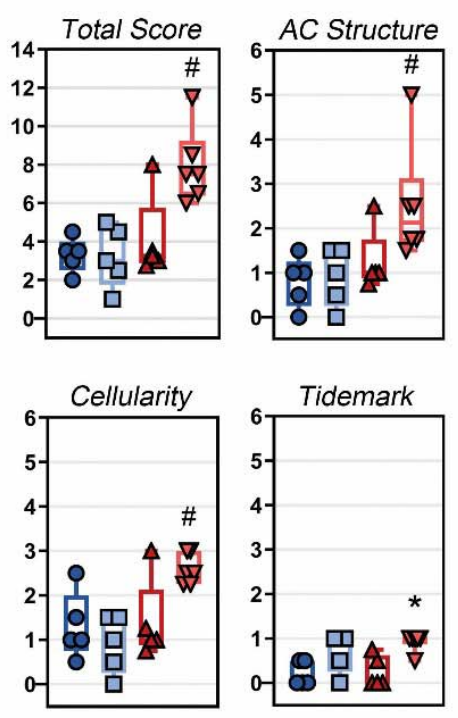

Sham Contralateral

Sham Uninjured

$\Delta$ ACLR Contralateral

$\nabla$ ACLR Injured
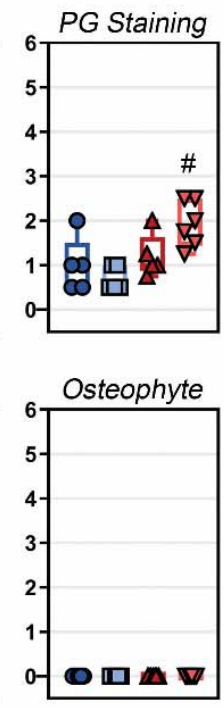

\section{Figure 4. Representative sagittal trochlea histology shows that ACLR Injured patellae exhibit}

mild superficial fissures (green arrowhead), and abnormal chondrocyte morphology, clustering, and focal regions of intense PG staining (red arrowheads) (A). ACLR Injured trochleae exhibit significantly higher OARSI Modified Mankin scores and sub-scores compared to Sham

Uninjured trochleae (Maximum OARSI scores: Total Score - 21; AC Structure - 8; PG Staining 
-6; Cellularity - 3; Tidemark-1; Osteophyte-3) (B). The top of the figure represents the

superior/proximal aspect of the trochlea. $n=6$ for all groups. * represents significant difference

compared to Contralateral limb $(P<0.05)$; ${ }^{*}$ represents significant difference compared to

Sham Uninjured limb $(P<0.05)$; Kruskal-Wallis ANOVA.

\section{DISCUSSION}

Significant articular cartilage and subchondral bone remodeling in the weight-bearing

regions of the tibiofemoral compartment are the hallmarks of PTOA in the knee joint. However, comparatively little is known about the development and progression of PTOA in the 
320

321

322

323

324

325

326

327

328

329

330

331

332

manifestations are indicative of early cartilage disease. Studies in both humans and animal models have shown that PTOA-associated AC degeneration initiates in the superficial zone with the development of surface irregularities, fissures, and disruption of the superficial collagen matrix ${ }^{17,18,39}$. Regarding cartilage thickness changes in response to injury, while some clinical studies of PTOA with early MRI assessment report compartment-specific regions of AC thickening ${ }^{14,27,29}$, other studies report short-term cartilage thickness loss that often persists into chronic disease $\mathrm{e}^{8,13,37}$. Previous studies of ACL rupture in rats have reported AC thickening in the tibial plateau, femoral condyles, and trochlea persisting for up to 10 weeks post-injury ${ }^{29}$, but to our knowledge this is the first study to report acute AC thickening in the patella using highresolution $\mu \mathrm{CT}$. In contrast to our findings, a recent study employing ACL transection in rats reported that both patellar and trochlear AC were histologically intact at 16 weeks post-injury, with surface degeneration and hypertrophic chondrocytes presenting by 32 weeks $^{40}$. While this may reflect inherent differences between surgical vs non-invasive ACL injury, our findings of early/mild cartilage damage underscore the value of high-resolution $\mu \mathrm{CT}$ in measuring the initial cartilage response to traumatic injury, which may be undetected and cannot be comprehensively quantified using histology or lower-resolution imaging modalities.

Acute loss of bone mineral density and bone volume following ACL rupture have been well-described in clinical literature ${ }^{22,41}$. Both clinical and pre-clinical studies have found that in the short term following ACL injury, subchondral and trabecular bone undergo catabolic degeneration, followed by a transition toward anabolic remodeling. In the subchondral bone, this remodeling persists in the long term, leading to subchondral bone sclerosis ${ }^{1,6,21}$. While animal models have accurately characterized this remodeling using high-resolution imaging, the exact nature of injury-induced catabolic bone remodeling in humans remains undescribed given that, to 
343 date, there have been very few clinical studies employing high-resolution imaging capable of

344 accurately measuring bone microarchitecture. Recently, however, Kroker et al used HR-pQCT to

345 demonstrate that femoral subchondral bone thickness and BMD decreases for 7-8 months post-

346 ACL injury before entering a plateau or recovery phase ${ }^{21}$. The subchondral and trabecular bone

347 results of the current study share key similarities with this clinical work, affirming that at 2-

348 weeks following ACLR, distinct bone remodeling-related indicators of early PFOA are readily

349 observable in this rat model.

A notable finding in our dataset is that several injury-induced changes occurred with

351 greater magnitude in the patella compared to changes in the trochlea. While a few prior clinical

352 studies have reported more severe cartilage loss in the patella compared to the femoral

353 condyles $^{37}$ and trochlea ${ }^{35}$, at least one other study has found that greater cartilage degeneration

354 occurs in the trochlea in the $2-5$ year post-injury time period ${ }^{8}$. Thus, it remains unclear to what

355 extent this finding is specific to this animal model or specific to a stage of PTOA severity, and it

356 is beyond the scope of the current study to investigate the cause of this difference. However,

357 previous clinical research has demonstrated that compared to the other articular surfaces of the

358 knee, patellar AC uniquely exhibits a differential compressive strain response to variations in

359 physical activity and intensity level, suggesting that the patella may be more sensitive to

360 mechanical stimuli and instability than the trochlea ${ }^{12,35}$. This is consistent with cadaveric

361 findings that patellar AC has higher fluid permeability and lower proteoglycan content than

362 femoral AC, potentially making the patella more susceptible to mechanically-induced

363 degeneration ${ }^{15}$. It is also possible that the mechanism of injury used in our model places a load

364 on the patella during compression and subluxation of the tibia. Given the location of the patella

365 relative to the trochlea in $100^{\circ}$ of flexion, this compression could be inducing some mild 
366

367

368

degeneration in the patella while having a lesser effect on the trochlea. Future studies may assess biomechanical and molecular mechanisms underpinning differential disease severity in the two surfaces of the patellofemoral compartment.

Some limitations should be taken into consideration when interpreting the results of this study. Although the Sham loading procedure did not cause any macroscopic injury or histologically-observable degeneration, it did have measurable effects on the AC and subchondral bone of the Sham limb compared to its contralateral. Sham effects are frequently reported in other models of rodent ACL injury, including patellar tendon ossification, increased $\mathrm{AC}$ water content, synovitis, and mild loss of proteoglycan at the AC surface resulting from a sham surgical procedure $e^{5,19,30,31}$. The non-invasive Sham procedure described here may have benefits over a surgical procedure involving skin and muscle incision and arthrotomy, which could introduce multiple biological confounding effects. Finally, due to the differences in histological processing and $\mu \mathrm{CT}$ scanning protocols used for the patella and the femur, we did not make any direct comparisons between the patella and trochlea.

In this rat model of non-invasive ACL rupture, we observed acute degeneration of the patellofemoral compartment, characterized by cartilage thickening and mild articular surface damage alongside catabolic trabecular and subchondral bone remodeling. Injury-induced changes in articular cartilage and trabecular bone were of greater severity in the patella than changes in the trochlea. These results demonstrate the acute onset of mild patellofemoral joint degeneration following ACL rupture and extend the utility of this rat joint injury model to the study of patellofemoral PTOA. 


\section{REFERENCES}

390

391

392

393

394

395

396

397

398

399

400

401

402

403

404

405

406

407

408

409

410

411

412

413

414

415

416

417

418

419

420

421

422

423

424

425

426

1. Anderson DD, Chubinskaya S, Guilak F, et al. Post-traumatic osteoarthritis: improved understanding and opportunities for early intervention. J Orthop Res. 2011;29(6):802-809.

2. Bouxsein ML, Boyd SK, Christiansen BA, et al. Guidelines for assessment of bone microstructure in rodents using micro-computed tomography. J Bone Miner Res. 2010;25(7):1468-1486.

3. Brown SB, Hornyak JA, Jungels RR, et al. Characterization of Post $\square$ Traumatic Osteoarthritis in Rats Following Anterior Cruciate Ligament Rupture by Non $\square$ Invasive Knee Injury (NIKI). J Orthop Res. 2019.

4. Carbone A, Rodeo S. Review of current understanding of post-traumatic osteoarthritis resulting from sports injuries. J Orthop Res. 2017;35(3):397-405.

5. Chou M-C, Tsai P-H, Huang G-S, et al. Correlation between the MR T2 value at 4.7 T and relative water content in articular cartilage in experimental osteoarthritis induced by ACL transection. Osteoarthritis Cartilage. 2009;17(4):441-447.

6. Christiansen BA, Anderson MJ, Lee CA, et al. Musculoskeletal changes following non-invasive knee injury using a novel mouse model of post-traumatic osteoarthritis. Osteoarthritis Cartilage. 2012;20(7):773-782.

7. Culvenor AG, Collins NJ, Guermazi A, et al. Early Patellofemoral Osteoarthritis Features One Year After Anterior Cruciate Ligament Reconstruction: Symptoms and Quality of Life at Three Years. Arthritis Care Res (Hoboken). 2016;68(6):784-792.

8. Culvenor AG, Eckstein F, Wirth W, Lohmander LS, Frobell R. Loss of patellofemoral cartilage thickness over 5 years following ACL injury depends on the initial treatment strategy: results from the KANON trial. Br J Sports Med. 2019;53(18):1168-1173.

9. Culvenor AG, Lai CC, Gabbe BJ, et al. Patellofemoral osteoarthritis is prevalent and associated with worse symptoms and function after hamstring tendon autograft ACL reconstruction. Br J Sports Med. 2014;48(6):435-439.

10. Culvenor AG, Segal NA, Guermazi A, et al. Sex $\square$ Specific influence of quadriceps weakness on worsening Patellofemoral and Tibiofemoral cartilage damage: a prospective cohort study. Arthritis Care Res. 2019;71(10):1360-1365.

11. Doube M, Kłosowski MM, Arganda-Carreras I, et al. BoneJ: free and extensible bone image analysis in ImageJ. Bone. 2010;47(6):1076-1079.

12. Eckstein F, Lemberger B, Gratzke C, et al. In vivo cartilage deformation after different types of activity and its dependence on physical training status. Ann Rheum Dis. 2005;64(2):291-295.

13. Eckstein F, Maschek S, Wirth W, et al. One year change of knee cartilage morphology in the first release of participants from the Osteoarthritis Initiative progression subcohort: association with sex, body mass index, symptoms and radiographic osteoarthritis status. Ann Rheum Dis. 2009;68(5):674-679.

14. Frobell RB. Change in cartilage thickness, posttraumatic bone marrow lesions, and joint fluid volumes after acute ACL disruption: a two-year prospective MRI study of sixty-one subjects. J Bone Joint Surg Am. 2011;93(12):1096-1103. 
15. Froimson MI, Ratcliffe A, Gardner TR, Mow VC. Differences in patellofemoral joint cartilage material properties and their significance to the etiology of cartilage surface fibrillation. Osteoarthritis Cartilage. 1997;5(6):377-386.

16. Gerwin N, Bendele A, Glasson S, Carlson CS. The OARSI histopathology initiativerecommendations for histological assessments of osteoarthritis in the rat. Osteoarthritis Cartilage. 2010;18:S24-S34.

17. Guilak F, Ratcliffe A, Lane N, Rosenwasser MP, Mow VC. Mechanical and biochemical changes in the superficial zone of articular cartilage in canine experimental osteoarthritis. J Orthop Res. 1994;12(4):474-484.

18. Hollander AP, Pidoux I, Reiner A, et al. Damage to type II collagen in aging and osteoarthritis starts at the articular surface, originates around chondrocytes, and extends into the cartilage with progressive degeneration. J Clin Invest. 1995;96(6):2859-2869.

19. Jackson MT, Moradi B, Zaki S, et al. Depletion of protease $\square$ activated receptor 2 but not protease $\square$ activated receptor 1 may confer protection against osteoarthritis in mice through extracartilaginous mechanisms. Arthritis Rheumatol. 2014;66(12):3337-3348.

20. Jarvela T, Paakkala T, Kannus P, Jarvinen M. The incidence of patellofemoral osteoarthritis and associated findings 7 years after anterior cruciate ligament reconstruction with bone-patellar tendon-bone autograft. Am J Sports Med. 2001;29(1):18-24.

21. Kroker A, Besler BA, Bhatla JL, et al. Longitudinal Effects of Acute Anterior Cruciate Ligament Tears on Peri-Articular Bone in Human Knees Within the First Year of Injury. J Orthop Res. 2019;37(11):2325-2336.

22. Kroker A, Bhatla JL, Emery CA, Manske SL, Boyd SK. Subchondral bone microarchitecture in ACL reconstructed knees of young women: A comparison with contralateral and uninjured control knees. Bone. 2018;111:1-8.

23. Record \#57 is using an undefined reference type. If you are sure you are using the correct reference type, the template for that type will need to be set up in this output style.

24. Lohmander LS, Ostenberg A, Englund M, Roos H. High prevalence of knee osteoarthritis, pain, and functional limitations in female soccer players twelve years after anterior cruciate ligament injury. Arthritis Rheum. 2004;50(10):3145-3152.

25. Maerz T, Kurdziel M, Newton MD, et al. Subchondral and epiphyseal bone remodeling following surgical transection and noninvasive rupture of the anterior cruciate ligament as models of post-traumatic osteoarthritis. Osteoarthritis Cartilage. 2016;24(4):698-708.

26. Maerz T, Kurdziel MD, Davidson AA, et al. Biomechanical characterization of a model of noninvasive, traumatic anterior cruciate ligament injury in the rat. Ann Biomed Eng. 2015;43(10):24672476.

27. Maerz T, Newton M, Matthew H, Baker K. Surface roughness and thickness analysis of contrastenhanced articular cartilage using mesh parameterization. Osteoarthritis Cartilage. 2016;24(2):290-298. 
28. Maerz T, Newton MD, Fleischer M, et al. Traumatic joint injury induces acute catabolic bone turnover concurrent with articular cartilage damage in a rat model of posttraumatic osteoarthritis. J Orthop Res. 2020.

29. Maerz T, Newton MD, Kurdziel MD, et al. Articular cartilage degeneration following anterior cruciate ligament injury: a comparison of surgical transection and noninvasive rupture as preclinical models of post-traumatic osteoarthritis. Osteoarthritis Cartilage. 2016;24(11):1918-1927.

30. Mapp P, Sagar D, Ashraf S, et al. Differences in structural and pain phenotypes in the sodium monoiodoacetate and meniscal transection models of osteoarthritis. Osteoarthritis Cartilage. 2013;21(9):1336-1345.

31. McErlain D, Appleton C, Litchfield R, et al. Study of subchondral bone adaptations in a rodent surgical model of OA using in vivo micro-computed tomography. Osteoarthritis Cartilage. 2008;16(4):458-469.

32. Neuman P, Kostogiannis I, Fridén T, et al. Patellofemoral osteoarthritis 15 years after anterior cruciate ligament injury-a prospective cohort study. Osteoarthritis Cartilage. 2009;17(3):284-290.

33. Newton MD, Junginger L, Maerz T. Automated MicroCT-based bone and articular cartilage analysis using iterative shape averaging and atlas-based registration. Bone. 2020:115417.

34. Oiestad BE, Holm I, Engebretsen L, et al. The prevalence of patellofemoral osteoarthritis 12 years after anterior cruciate ligament reconstruction. Knee Surg Sports Traumatol Arthrosc. 2013;21(4):942-949.

35. Owusu-Akyaw KA, Heckelman LN, Cutcliffe HC, et al. A comparison of patellofemoral cartilage morphology and deformation in anterior cruciate ligament deficient versus uninjured knees. J Biomech. 2018;67:78-83.

36. Palmer AW, Guldberg RE, Levenston ME. Analysis of cartilage matrix fixed charge density and three-dimensional morphology via contrast-enhanced microcomputed tomography. Proc Natl Acad Sci U S A. 2006;103(51):19255-19260.

37. Potter HG, Jain SK, Ma Y, et al. Cartilage injury after acute, isolated anterior cruciate ligament tear: immediate and longitudinal effect with clinical/MRI follow-up. Am J Sports Med. 2012;40(2):276285.

38. Ramme A, Lendhey M, Raya J, Kirsch T, Kennedy O. A novel rat model for subchondral microdamage in acute knee injury: a potential mechanism in post-traumatic osteoarthritis. Osteoarthritis Cartilage. 2016;24(10):1776-1785.

39. Stoop R, Buma P, van der Kraan PM, et al. Type II collagen degradation in articular cartilage fibrillation after anterior cruciate ligament transection in rats. Osteoarthritis Cartilage. 2001;9(4):308-315.

40. Tsai PH, Lee HS, Siow TY, et al. Abnormal perfusion in patellofemoral subchondral bone marrow in the rat anterior cruciate ligament transection model of post-traumatic osteoarthritis: a dynamic contrast-enhanced magnetic resonance imaging study. Osteoarthritis Cartilage. 2016;24(1):129-133.

41. van Meer BL, Waarsing JH, van Eijsden WA, et al. Bone mineral density changes in the knee following anterior cruciate ligament rupture. Osteoarthritis Cartilage. 2014;22(1):154-161. 